\title{
ESTRATÉGIA ALIMENTAR DO ESPADARTE (Xiphias gladius) NO ATLÂNTICO EQUATORIAL SUDOESTE
}

\author{
Teodoro VASKE JÚNIOR \\ Rosângela Paula LESSA
}

\author{
DIMAR (Laboratório de Dinâmica de Populações Marinhas) \\ Departamento de Pesca, Universidade Federal Rural de \\ Pernambuco (UFRPE) \\ Av. Dom Manuel de Medeiros s/n, Dois Irmãos \\ Recife PE \\ CEP: $52171-900$ \\ Tel: 081-33021518 \\ e-mail: vaske@ig.com.br
}

Recebido: $16 / 08 / 2005$

Aceito: 29/08/2005

\section{RESUMO}

O espadarte, Xiphias gladius, é um predador de importância comercial que chega a representar 50 \% das capturas com espinhel de superfície no Atlântico equatorial sudoeste. Sua estratégia alimentar foi estudada nos aspectos de composição da dieta e relações presa-predador. Foram analisados um total de 231 estômagos, dos quais 193 apresentavam conteúdo. Foram identificados 39 itens alimentares, onde de acordo com o Índice de Relativa Importância (IRI), os peixes Brama caribbea, Selar crumenophtalmus, Gempylus serpens, o cefalópode Ommastrephes bartramii, e o camarão Heterocarpus ensifer foram os principais itens alimentares. Não houve variação do tamanho das presas com o tamanho do predador, o tamanho médio das presas foi de 10,2 cm. A maioria dos estômagos apresentou entre 1 e 5 presas com um máximo de 36 . O espadarte preda preferencialmente organismos do epipelágico fazendo incursões ocasionais a grandes profundidades para predar lulas e peixes do mesopelágico.

Palavras-chave: Espadarte, Xiphias gladius, alimentação

\section{ABSTRACT \\ (FEEDING STRATEGY OF THE SWORDFISH (Xiphias gladius) IN THE SOUTHWESTERN EQUATORIAL ATLANTIC)}

The swordfish, Xiphias gladius, is a predador of commercial importance that sometimes represent $50 \%$ of total catches in longline fishery from Southwestern equatorial Atlantic. The feeding strategy was studied in the aspects of diet composition and predator-prey relationships. A total of 231 stomachs were analized, of which 193 presented some food. Thirty nine food items were identified. According IRI ranking, the fishes Brama caribbea, Selar crumenophtalmus, Gempylus serpens, the cephalopod Ommastrephes bartramii, and the shrimp Heterocarpus ensifer were the main food items. No variations were found between prey length and predator length, where preys presented $10.2 \mathrm{~cm}$ mean length. Most stomachs presented 1 to 5 preys with a maximum of 36. The swordfish prey preferencially upon epipelagic organisms, making occasional incursions at great depths to prey mesopelagic fishes and squids.

Key words: Swordfish, Xiphias gladius, alimentação

Tropical Oceanography, Recife, v. 33, n. 2, p. 223-232, 2005. 


\section{INTRODUÇÃO}

O espadarte, Xiphias gladius, é um predador pelágico com distribuição entre $45^{\circ} \mathrm{N}$ e $45^{\circ} \mathrm{S}$ em todos os oceanos e mares tropicais e subtropicais (Palko et al., 1981). A sua distribuição vertical vai da superfície aos $600 \mathrm{~m}$ em águas entre 5 e $27^{\circ} \mathrm{C}$ (Nakamura, 1985). No Brasil é capturado regularmente em toda costa com espinhel de superfície. Mais especificamente na região nordeste, o espadarte representou em torno de $6 \%$ das capturas totais de pesca de espinhel entre 1983 e 1997, passando a ter um considerável aumento nas cotas a partir de 1998, chegando a representar $50 \%$ do total desembarcado (Hazin et al., 1999).

Estudos de alimentação de espadarte têm sido realizados no Atlântico Norte com enfoque principal nas listas de presas e proporções de presas na dieta (Tibbo et al., 1961; Scott \& Tibbo, 1968, 1974; Stillwell \& Kohler, 1985; Hernández-Garcia, 1995; Simões e Andrade, 1999). No Brasil, os estudos mais importantes foram realizados por Zavala-Camin (1981) no sudeste e sul com destaque para as presas peixe e por Mello (1992) no sul enfocando espectro e intensidade alimentar. Não há trabalhos de alimentação de espadarte para a região sudoeste equatorial do Atlântico, por isso o presente estudo objetiva conhecer o espectro alimentar e as relações presa-predador do espadarte capturado no nordeste do Brasil, como subsídio para estudos posteriores de relações tróficas nesta região.

\section{MATERIAL E MÉTODOS}

As amostras foram obtidas de embarques científicos do NPq Riobaldo (CEPENE-IBAMA), entre 1993 e 2001, na área oceânica do nordeste brasileiro incluindo as adjacências do Atol das Rocas e Arquipélago de Fernando de Noronha (Fig. 1). A arte de pesca utilizada foi o espinhel de superfície multifilamento, modelo Suzuki et al. (1977), com 600 anzóis, e samburás de 6 e de 12 anzóis.

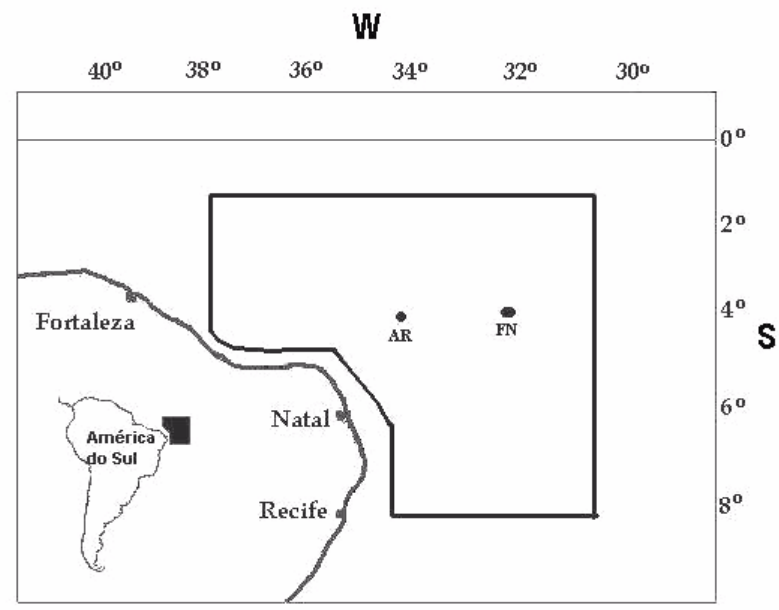

Figura 1 - Área de amostragem de Xiphias gladius no nordeste do Brasil. AR - Atol das Rocas, FN - Arquipélago de Fernando de Noronha.

Os estômagos foram coletados durante a evisceração logo após o desembarque dos peixes, sendo seccionados e amarrados na porção do esôfago e intestino delgado. A seguir recebiam uma injeção de formalina 5\%, etiqueta com dados de coleta, e eram acondicionados em sacos plásticos furados e imersos em tonéis com formalina 5\%. Dos espadartes foram tomados dados de 
comprimento furcal (extremidade da mandíbula inferior até o ponto médio da cauda) em centímetros, data e local de captura.

Foi considerado o conteúdo estomacal de todo material retido em peneira de $1 \mathrm{~mm}$ de malha. As categorias de presas foram chamadas de "item alimentar". Por sua vez, cada unidade de item alimentar foi chamada de "presa", de onde foram tomados os comprimentos totais em centímetros e peso em gramas, quando possível. Para os cefalópodes foi medido o comprimento do manto em centímetros. Os itens alimentares foram identificados ao menor táxon possível.

A importância de cada item alimentar foi obtida através do Índice de Relativa Importância (IRI) modificado para peso (Pinkas et al., 1971; Amezaga-Herrán, 1988):

onde:

$$
\mathrm{IRI}_{\mathrm{i}}=\% \mathrm{FO}_{\mathrm{i}} \mathrm{x}\left(\% \mathrm{~N}_{\mathrm{i}}+\% \mathrm{P}_{\mathrm{i}}\right)
$$

\% $\mathrm{FO}_{\mathrm{i}}$ - porcentagem da freqüência de ocorrência de cada item alimentar

$\% \mathrm{~N}_{\mathrm{i}}$ - porcentagem em número de presas ingeridas de cada item alimentar

$\% \mathrm{P}_{\mathrm{i}}$ - porcentagem em peso de presas ingeridas de cada item alimentar

Para o cálculo de IRI não foram considerados como conteúdo alimentar os bicos isolados e sem massa muscular dos cefalópodes, evitando-se assim uma sobrestimativa em número deste grupo. Devido a um eventual acúmulo de bicos nos estômagos oriundos de alimentação de horas ou de dias anteriores, não se pode determinar a quanto tempo o cefalópode foi predado e digerido (Vaske \& Rincón, 1998).

\section{RESULTADOS}

Os comprimentos dos peixes variaram entre 58 e $290 \mathrm{~cm}$, com média de 162,6 cm (Fig. 2). Houve capturas proporcionais de indivíduos maturos e imaturos, uma vez que o espadarte atinge a maturidade sexual em torno de $155 \mathrm{~cm}$ (Hazin et al., 1999).

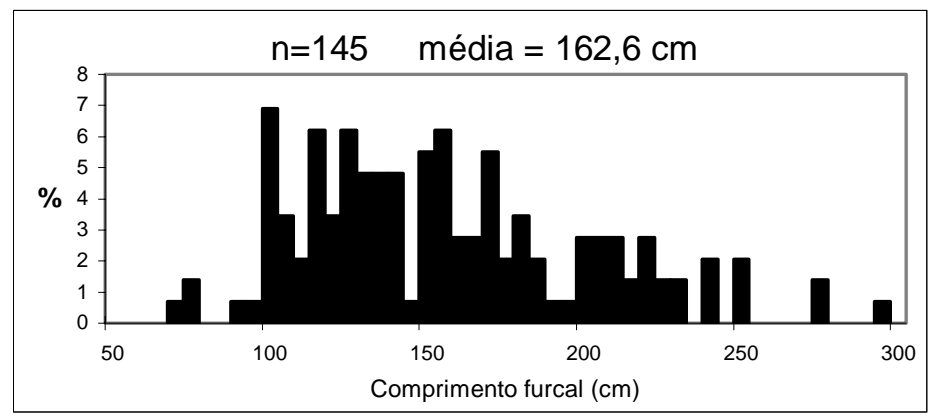

Figura 2 - Distribuição de comprimentos de Xiphias gladius no nordeste do Brasil.

Foram analisados 231 estômagos, dos quais 193 apresentaram conteúdo. Com aproximadamente 150 estômagos analisados observou-se uma estabilização na riqueza de itens alimentares o que evidencia que o espectro trófico foi adequadamente amostrado (Fig. 3). 


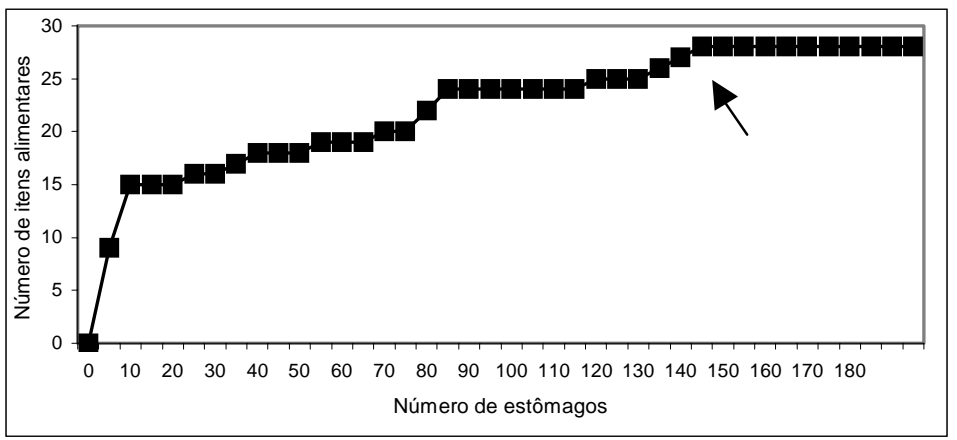

Figura 3 - Relação entre número de itens alimentares e estômagos analisados para Xiphias gladius. A seta indica o início da estabilização.

Foram identificados 39 itens alimentares, com amplo predomínio de peixes e cefalópodes, e ainda ocorrências de crustáceos e gastrópodes (Tabela 1). Se forem considerados os itens ao nível de espécie, observa-se que os peixes Brama caribbea, Selar crumenophtalmus, Gempylus serpens, o cefalópode Ommastrephes bartramii, e o camarão Heterocarpus ensifer são os principais itens alimentares do espadarte na região estudada. O item Teleostei representa peixes teleósteos não identificados, tendo sua importância no cálculo do montante que os peixes ósseos representam na dieta.

Tabela 1 - Porcentagens em número (\%N), peso (\%P) e freqüência de ocorrência (\%FO) dos itens alimentares de Xiphias gladius Estômagos com conteúdo: 193

Estômagos vazios: 38

\begin{tabular}{|c|c|c|c|}
\hline PRESAS & $\mathbf{N}$ & $\% \mathrm{~N}$ & $\mathbf{P}$ \\
\hline Teleostei & 184 & 24,53 & 5651 \\
\hline Brama caribbea & 140 & 18,67 & 4065 \\
\hline Selar crumenophtalmus & 58 & 7,733 & 4320 \\
\hline Gempylus serpens & 18 & 2,4 & 1056 \\
\hline Scombridae & 11 & 1,467 & 803 \\
\hline Aluterus sp. & 10 & 1,333 & 1833 \\
\hline Diodon hystrix & 13 & 1,733 & 127 \\
\hline Cypselurus spp. & 6 & 0,8 & 233 \\
\hline Dactylopterus volitans & 5 & 0,667 & 5 \\
\hline Alepisaurus ferox & 1 & 0,133 & 339 \\
\hline Acanthurus sp. & 6 & 0,8 & 4 \\
\hline Decapterus punctatus & 2 & 0,267 & 93 \\
\hline Ruvettus pretiosus & 2 & 0,267 & 70 \\
\hline Caranx spp. & 2 & 0,267 & 50 \\
\hline Balistes vetula & 2 & 0,267 & 36 \\
\hline Coryphaena hippurus & 1 & 0,133 & 28 \\
\hline Diaphus sp. & 1 & 0,133 & 7 \\
\hline
\end{tabular}


Tabela 1 - Porcentagens em número $(\% \mathrm{~N})$, peso $(\% \mathrm{P})$ e freqüência de ocorrência (\%FO) dos itens alimentares de Xiphias gladius

\begin{tabular}{|c|c|c|c|}
\hline PRESAS & $\mathbf{N}$ & $\% \mathrm{~N}$ & $\mathbf{P}$ \\
\hline PEIXES & 462 & 61,6 & 187 \\
\hline Ommastrephes bartramii & 42 & 5,6 & 169 \\
\hline Histioteuthis spp. & 39 & 5,2 & 21 \\
\hline Histioteuthis spp.(bico) & 32 & 4,267 & \\
\hline Cephalopoda & 15 & 2 & 24 \\
\hline Japetella diaphana (bico) & 27 & 3,6 & \\
\hline Hyaloteuthis pelagica (bico) & 29 & 3,867 & \\
\hline Cranchiidae & 13 & 1,733 & 27 \\
\hline Onychoteuthidae & 7 & 0,933 & 24 \\
\hline Ornithoteuthis antillarum (bico) & 13 & 1,733 & \\
\hline Ommastrephes bartramii (bico) & 15 & 2 & \\
\hline Ornithoteuthis antillarum & 14 & 1,867 & 27 \\
\hline Hyaloteuthis pelagica & 20 & 2,667 & 10 \\
\hline Onychoteuthidae (bico) & 6 & 0,8 & \\
\hline Cranchiidae (bico) & 2 & 0,267 & \\
\hline Ommastrephidae (bico) & 2 & 0,267 & \\
\hline Sthenoteuthis pteropus (bico) & 2 & 0,267 & \\
\hline Ommastrephidae & 1 & 0,133 & 51 \\
\hline Octopodidae & 1 & 0,133 & 40 \\
\hline Sthenotethis pteropus & 2 & 0,267 & 3 \\
\hline Thysanoteuthis rombus & 1 & 0,133 & 12 \\
\hline Chiroteuthis sp. & 1 & 0,133 & 2 \\
\hline Octopoteuthis sp. & 1 & 0,133 & 2 \\
\hline Ocythoe tuberculata & 1 & 0,133 & 2 \\
\hline Tremoctopus violaceus & 1 & 0,133 & 2 \\
\hline Chiroteuthis sp. (bico) & 1 & 0,133 & \\
\hline Thysanoteuthis rombus (bico) & 1 & 0,133 & \\
\hline CEFALÓPODES (apenas bicos) & 129 & 17,2 & \\
\hline CEFALÓPODES & 160 & 21,33 & 281 \\
\hline Heterocarpus ensifer & 74 & 9,867 & 20 \\
\hline Brachyura & 7 & 0,933 & 2 \\
\hline Isopoda & 3 & 0,4 & 3 \\
\hline Nephropidae & 1 & 0,133 & 1 \\
\hline Squiliidae & 1 & 0,133 & 1 \\
\hline CRUSTÁCEOS & 86 & 11,47 & 20 \\
\hline Bursa sp. & 42 & 5,6 & 5 \\
\hline GASTRÓPODES & 42 & 5,6 & 5 \\
\hline TOTAL & 750 & 100 & 217 \\
\hline
\end{tabular}

A representatividade dos cefalópodes na dieta variou conforme a consideração dos bicos na contabilidade. Os cefalópodes identificados apenas pelos bicos representaram 44,6 \% do total de cefalópodes e foram considerados no conhecimento da diversidade dos itens alimentares.

O comprimento das presas variou de 2 a $65 \mathrm{~cm}$, embora a maior quantidade observada tenha se situado entre 2 e $20 \mathrm{~cm}$, com média geral de 11,3 cm (Fig. 4). 


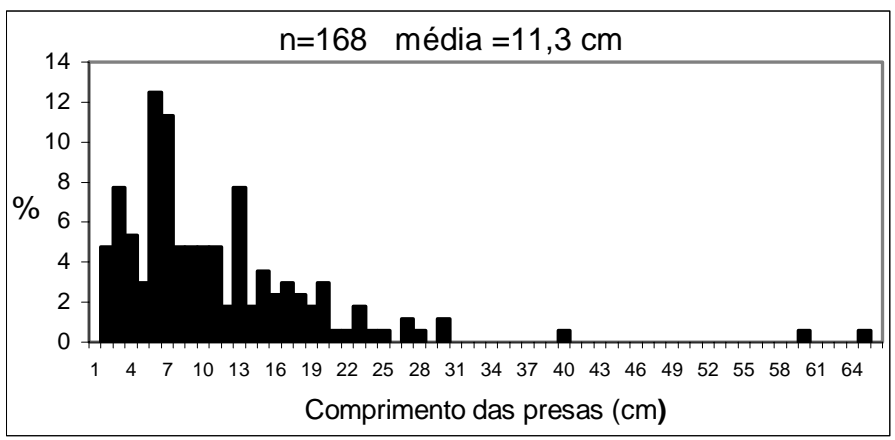

Figura 4 - Comprimento das presas de Xiphias gladius.

Não houve variação do tamanho das presas em relação ao comprimento do predador, sendo notável a constância de tamanhos de presas entre as classes de 110 e $290 \mathrm{~cm}$, com uma média geral de 10,2 cm (Fig. 5).

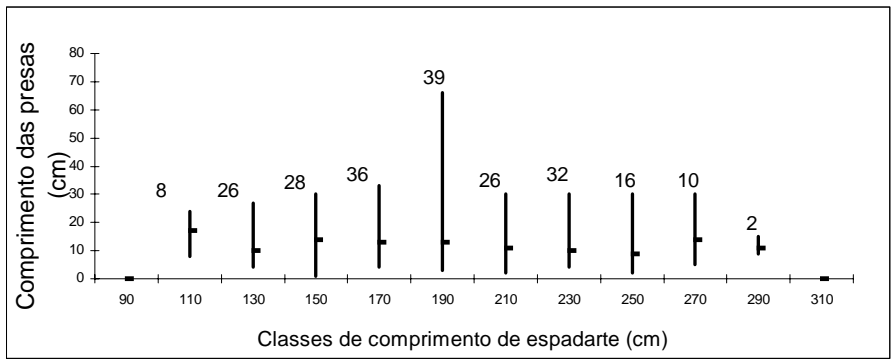

Figura 5 - Amplitudes e comprimentos médios das presas para diferentes classes de comprimento de Xiphias gladius. O valor no topo das barras refere-se ao número de presas medidas.

Cabe ressaltar que os comprimentos dos cefalópodes, quando incluídos os tentáculos, podem chegar a cinco vezes o comprimento do manto como em Chiroteuthis sp., no entanto, os tentáculos não foram considerados devido a pouca massa muscular em relação ao corpo. As presas de maiores comprimentos foram todas observados em exemplares da classe de $190 \mathrm{~cm}$, onde a maior foi de um exemplar do peixe Gempylus serpens com $65 \mathrm{~cm}$. A grande maioria dos estômagos apresentou entre 1 e 10 presas por estômago, com predominância de ocorrência de apenas uma unidade por estômago (Fig. 6). 


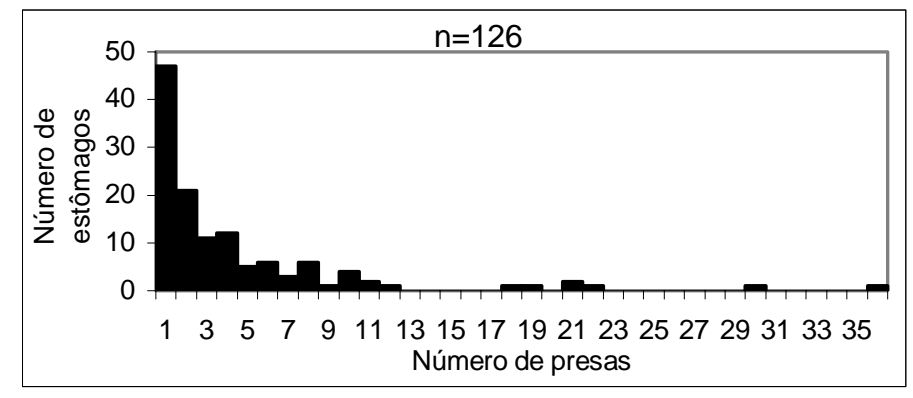

Figura 6 - Número de presas por estômago de Xiphias gladius.

O peso do conteúdo estomacal mostrou uma leve tendência de diminuição de proporção com o aumento do comprimento ou peso do predador, ou seja, indivíduos menores que $150 \mathrm{~cm}$ tendem a ter proporcionalmente mais alimento nos estômagos (Fig. 7).

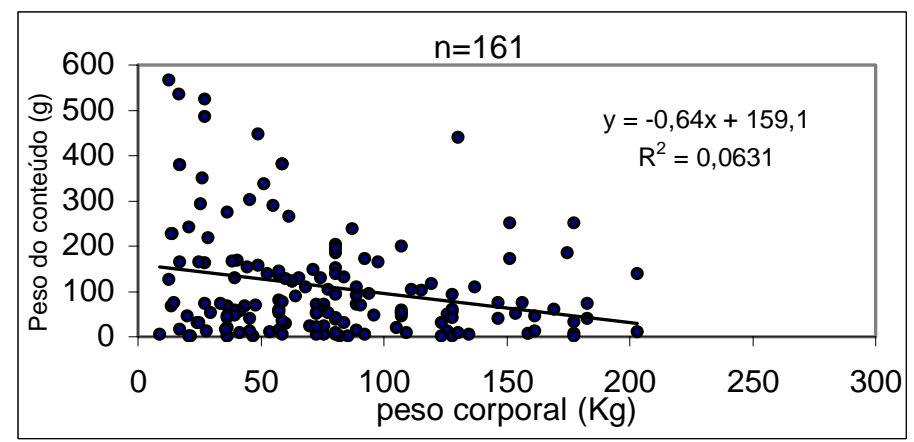

Figura 7 - Relação entre o peso do conteúdo estomacal e o peso corporal de Xiphias gladius.

\section{DISCUSSÃO}

Além da ampla distribuição horizontal em todos os oceanos, o espadarte faz ocasionalmente incursões em águas mais profundas, chegando a $600 \mathrm{~m}$ conforme verificado por telemetria acústica (Carey \& Robinson, 1981; Carey, 1982), embora a maior parte do tempo permaneça no epipelágico. Segundo Lokkenborg \& Byordal (1992), quanto maior o tamanho corporal do predador, maior o deslocamento e maior a área varrida a procura de alimento, aí se enquadram os grandes predadores como o espadarte que se deslocam a procura de alimento e tem portanto, maior probabilidade de contato com artes passivas como o espinhel e rede de emalhe. Esta capacidade de explorar amplas regiões a procura de alimento é possível em função dos tamanho dos olhos e conservação de calor corporal permitindo que prede organismos que permanecem nas profundidades menos iluminadas e mais frias onde outros predadores têm dificuldade de localização visual (Carey, 1992). No presente estudo isto pôde ser constatado pela presença de cefalópodes mesopelágicos como Cranchiidae, Chiroteuthis sp., Histioteuthis sp. e Japetella diaphana nos estômagos. Entre os 23 taxa de cefalópodes, quase todos fazem migração vertical permanecendo no epipelágico durante a noite e migrando ao mesopelagial durante o dia com exceção dos polvos Tremoctopus. violaceus e Ocythoe tuberculata que permanecem sempre no epipelágico (Roper \& 
Young, 1975). Com exceção do peixe mesopelágico Alepisaurus ferox, todos os outros peixes são representantes do epipelágico, de onde se pode concluir que o espadarte procura se alimentar de peixes nas camadas superficiais e ocasionalmente faz incursões em camadas mais profundas a procura de cefalópodes, uma vez que cefalópodes e peixes mesopelágicos têm pouca mobilidade, com estrutura corporal frágil e gelatinosa, e oferecem outra opção alimentar que exige menor gasto energético na captura. Segundo Zavala-Camin (1981), os cefalópodes representaram 68,1\% em freqüência de ocorrência da dieta para o espadarte no sudeste e sul do Brasil. Posteriormente, Mello (1992) observou entre 77 e 79\% de participação em número e entre 38 e 55\% de participação em peso de cefalópodes nas dietas de espadarte no sul do Brasil. Bello (1991) salienta a importância dos cefalópodes nos conteúdos de espadarte no Mediterrâneo por representarem 85 \% de freqüência de ocorrência dos conteúdos. No presente estudo, os peixes foram mais representativos na dieta provavelmente por ser esta região equatorial pouco abundante em grandes biomassas de cefalópodes.

A influência dos bancos oceânicos, Atol das Rocas e Arquipélago de Fernando de Noronha foi a causa provável da ocorrência de parte dos organismos do micronécton na alimentação, particularmente os peixes brefoepipelágicos das famílias Dactylopteridae, Carangidae, Diodontidae e Holocentridae, cujos adultos habitam as águas costeiras das ilhas. O peixe xixarro (Selar crumenophtalmus) ocorreu somente em estômagos oriundos da proximidade ao talude de Pernambuco; o que pode indicar predação em cardumes desta espécie no talude. Nakamura (1985) comenta que os espadartes que estão próximos a regiões costeiras preferem se dirigir às espécies mais neríticas do que as da área oceânica adjacente. Palko et al. (1981) observam que o espadarte come o que quer que esteja em grande quantidade no seu ambiente, baseando-se na lista de presas de oito publicações entre 1949 e 1970 no Atlântico e Pacífico. Na área oceânica próxima ao nordeste do Brasil, Ovchinikov (1970) ressalta que as presas mais importantes do espadarte foram Teuthoidea, Vomer sp., Octopoda e Exocoetidae.

A ocorrência de pequenos organismos como megalopas de Brachyura (entre 1 e $2 \mathrm{~cm}$ de comprimento) pode ser considerada como acidental uma vez que o espadarte não possui rastros branquiais. A exceção é o camarão mesopelágico Heterocarpus ensifer de maior porte $(5-10 \mathrm{~cm})$ e também do gastrópode Bursa sp. ingeridos nas proximidades do talude do Estado de Pernambuco.

A maior quantidade de estômagos com algumas unidades de presas é coerente com a maioria dos estômagos com conteúdos pesando menos de $200 \mathrm{~g}$, o que é um indício de que o espadarte se alimenta constantemente e em pequenas quantidades por vez. Provavelmente este ritmo alimentar esteja relacionado com a distribuição das presas, que nessa região equatorial oligotrófica, estão espalhadas e em baixas densidades, obrigando os predadores a uma constante busca e ingestão de alimento para suprir o gasto energético das migrações.

\section{REFERÊNCIAS BIBLIOGRÁFICAS}

AMEZAGA-HERRÁN, R. 1988. Analisis de contenidos estomacales en peces. Revision bibliografica de los objetivos y la metedologia. Inf. Tec. Inst. Esp. Oceanogr., N.o 63, 74 p.

BELLO, G. 1991. Role of cephalopods in the diet of the swordfish, Xiphias gladius, from the eastern Mediterranean. Sea Bull. Mar. Sci. (49)(1-2):312-324.

CAREY, F.G. 1992. Through the thermocline and back again. Heat regulation in big fish. Oceanus 35(3): 79-85.

CAREY F.G.\& B.H.ROBINSON. 1981 Daily patterns In the activities of swordfish, Xiphias gladius observed by acoustic telemetry. Fish. Bull. 79(2):277-292.

Tropical Oceanography, Recife, v. 33, n. 2, p. 223-232, 2005. 
HAZIN, F .H. V.; H. G. HAZIN; C. E. BOECKMANN; P. TRAVASSOS; C. MARQUES. 1999. Structure de la populacion de l'espadon (Xiphias gladius) dans l'Atlantique Sud-ouest Équatorial. Col. Vol. Sci. Pap. ICCAT, 52:1226-1232.

HERNÁNDES-GARCIA, V. 1995. The diet of the swordfish Xiphias gladius Linnaeus, 1758, in the central east Atlantic with emphasis on the role of cephalopods. Fish. Bull. 93:4O3-411.

LOKKEBORG, S. and A BYORDAL. 1992. Species and size selectivity in longline fishing: a review. Fishery Research, 13:311-322.

MELLO, R.M.1992. Análise de conteúdos estomacais, intensidade de alimentação, idade e crescimento do espadarte, Xiphias gladius (Xiphioidei:Xiphiidae), no sul do Brasil. Tese de mestrado. Fundação Univrsidade Federal do Rio Grande. 105 p.

NAKAMURA, I. 1985. FAO Species catalogue. Vol. 5. Billfishes of the World. An annotated and illustrated catalogue of marlins, sailfishes, spearfishes and swordfishes know to date. FAO Fish. Synop. (125) Vol.5: 65 p.

OVCHINIKOV, V.V. 1970. Swordfishes and billfishes in the Atlantic ocean. Ecology and functional morphology. Nauch-Isled. Inst. Ryb. Khoz. Okeanogr. Kaliningrad.:106 p. (Translated from russian by Israeli Prog. Sci.).

PALKO, B.J., G.L.BEARDSLEY and W.J. RICHARDS. 1981. Synopsis of the biology of the swordfish, Xiphias gladius Linnaeus. NOAA, NMFS Tech. Rep. 441, FAO Fish. Syn. N.o 127.

PINKAS, L.; M.S. OLIPHANT; I.L.K. IVERSON. 1971. Food habits of albacore, bluefin tuna, and bonito in Californian waters. Calif. Dep. Fish Game, Fish. Bull. 152:105 p.

ROPER, C.F.E. and R.E. YOUNG. 1975. Vertical distribution of pelagic cephalopods. Smithson.

Contrib. Zool. No. 209: 1-51.

SCOTT , W. B. \& S.N.TIBBO. 1968. Food and feeding habits of swordfish, Xiphias gladius, in the Western North Atlantic. J. Fish. Res. Bd. Can. 26(5):903-919.

SCOTT , W. B. \& S.N.TIBBO. 1974. Food and feeding habits of swordfish, Xiphias gladius, Linnaeus, in the Northwest Atlantic ocean. NOAA Tech Rep. 675:138-141.

SIMÕES, P. R. \& J. P. ANDRADE. 1999. Feeding dynamics of swordfish (Xiphias gladius) in Azores area. ICCAT/ SCRS. Manuscript. 15 p.

STILLWELL, C.E. \& N.E. KOHLER. 1985. Food and feeding ecology of the sworfish Xiphias gladius In the Western North Atlantic with estimates of daily ration. Mar. Ecol. Vol 22:239-247.

SUZUKI, Z.: WARASHINA, Y. and M. KISHIDA, 1977. The comparison of catches by regular and deep tuna longline gears in the western and central equatorial Pacific. Bull. Far Seas Fish. Res. Lab., 15:51-89.

TIBBO, S.N.; L. R. DAY; W. F. DOUCET. 1961. The swordfish (Xiphias gladius L.) its life-history and economic importance in the Northwest Atlantic. J. Fish. Res. Bd. Can., 130, 47 p.

Tropical Oceanography, Recife, v. 33, n. 2, p. 223-232, 2005. 
VASKE JR, T.; G.Fo. RINCÓN. 1998. Conteúdo estomacal dos tubarões azul (Prionace glauca) e anequim (Isurus oxyrinchus) em águas oceânicas no sul do Brasil. Rev. Bras. Biol. Vol 58 (3):443450 .

ZAVALA-CAMIN, L.A., 1981. Hábitos alimentares e distribuição dos atuns e afins (Osteichthyes Teleostei) e suas relações ecológicas com outras espécies pelágicas das regiões sudeste e sul do Brasil. Tese de doutorado, Instituto de Biociências da Universidade de São Paulo, Brasil. 237 pp. 